\title{
MediBot: Um chatbot para consulta de riscos e informações sobre medicamentos
}

\author{
Caio Viktor S. Avila, Tulio Vidal Rolim, \\ José Wellington Franco da Silva, Vania Maria Ponte Vidal \\ ${ }^{1}$ Departamento de Computação - Universidade Federal do Ceará (UFC) \\ Fortaleza, CE - Brazil \\ caioviktor@alu.ufc.br, tulio.xcrtf@gmail.com \\ wellington@crateus.ufc.br, vvidal@lia.ufc.br
}

\begin{abstract}
Self-medication without medical advice can lead to health problems through intoxication. The objective of this study is to present MediBot - a chatbot to consult information about medications and their risks. MediBot enables queries through natural language, transformed them into SPARQL queries over a Linked Data Mashup about data on medicines provided by ANVISA and Sider sources. Finally, MediBot presents itself as a timely tool in promoting access to information by the general public.
\end{abstract}

Resumo. A automedicação sem orientação médica pode vir a acarretar problemas de saúde por meio da intoxicação.O objetivo deste estudo consiste em apresentar MediBot - um chatbot para consulta de informações sobre medicamentos e seus riscos. O MediBot permite a realização de consultas através de linguagem natural, transformado-as em consultas SPARQL sobre um Linked Data Mashup envolvendo dados sobre medicamentos providos pelas fontes ANVISA e Sider. Por fim, MediBot apresenta-se como instrumento oportuno na promoção do acesso à informação por parte do público geral.

\section{Introdução}

O uso de medicamentos para tratamento de doenças e enfermidades é percebido como uma prática válida para melhorias no que tange ao estado de saúde da população. Contudo, a ingestão inadequada de medicamentos pode acarretar a ocorrência de efeitos colaterais e intoxicação. Segundo a Organização Mundial da Saúde (OMS) [WHO et al. 2002], são definidos como eventos adversos à medicamentos quaisquer ocorrências médicas desfavoráveis que possam ocorrer durante a fase de tratamento medicamentoso.

No Brasil, entre os agentes causadores de intoxicação, como agrotóxicos, drogas ilícitas, rodenticidas, inseticidas e alimentos impróprios para consumo, os medicamentos ocupam a primeira posição [Corrêa et al. 2013]. Somente em 2016, houveram aproximadamente 56.937 casos registrados de intoxicação humana, dos quais, 226 evoluíram para óbito, sendo 20.527 gerados em decorrência do uso de medicamentos [SINITOX 2016].

$\mathrm{Na} w e b$, existe uma grande variedade de dados sobre medicamentos que podem auxiliar na orientação de uma medicação correta, tais dados são provenientes de organizações governamentais, como agências reguladoras e portais de dados públicos, fabricantes, farmácias on-line e boletins eletrônicos. Contudo, a grande maioria destes 
dados está em formato proprietário, como planilhas, em backups de bancos de dados relacionais ou disponíveis apenas por meio de páginas da web. Além disso, esses dados são isolados em silos de dados, sem conexão direta entre recursos, onde por vezes, esses datasets são representados com diferentes vocabulários, tendo um mesmo conceito do mundo real representado através de termos distintos. Um exemplo de informação que não pode ser recuperada diretamente é dada a seguir: "Quais são os riscos do medicamento XX?".

Apesar de ser uma consulta simples, apresenta alguns desafios como: heterogeneidade das fontes (dados em rdf, csv, xls e etc) e a necessidade do conhecimento prévio dos usuários sobre tecnologias e domínio em questão. Esses problemas podem ser resolvidos usando tecnologias da Web Semântica[Shadbolt et al. 2006] e Linked Data [Bizer et al. 2011] que permitem uma integração semântica entre recursos em diferentes fontes, representando os dados em um vocabulário unificado através do uso de ontologias. A ontologia fornece um vocabulário uniforme, atuando como uma camada de abstração para acesso a dados. Além disso, permite sua publicação em um formato aberto não proprietário que permite a recuperação de informações por agentes computacionais e usuários humanos através de consultas SPARQL.

Este trabalho tem como objetivo demostrar como MediBot, um chatbot baseado em ontologia, pode ser utilizado como ferramenta de acesso à dados sobre medicamentos e seus riscos.

O resto deste artigo é estruturado da seguinte forma: na seção 2 é apresentada a ferramenta MediBot. A seção 3 apresenta a arquitetura de MediBot. Na seção 4 é apresentada a demonstração proposta. Por fim, na seção 5 são apresentadas nossas considerações finais e trabalhos futuros.

\section{MediBot}

MediBot é um chatbot que pode ser utilizado tanto através do aplicativo móvel do Telegram e de sua interface web, quanto no ambiente desktop. MediBot foi implementado em JavaScript utilizando NodeJS. MediBot pode ser contactado via Telegram através do id @LDM_MediBot. Para mais informações, vídeos demonstrativos e exemplos acessar o link $^{1}$.

MediBot possui dois modos de funcionamento, sendo o primeiro o modo de respostas rápidas e o segundo o interativo. No modo de respostas rápidas, MediBot possui um conjunto de consultas $S P A R Q L$ pré-definidas para as quais a entrada do usuário é mapeada. O mapeamento da entrada do usuário para o seu respectivo padrão de consulta é realizado através de uma abordagem baseada em expressões regulares. Durante o processo de avaliação da entrada, os termos-chave e os parâmetros de filtragem são recuperados. Os termos-chave ajudam a classificar em qual tipo de consulta a entrada deve ser mapeada, enquanto os parâmetros de filtragem são usados em cláusulas FILTER para restringir o resultado da consulta à intenção específica do usuário. Finalmente, a consulta SPARQL é construída e executada via HTTP no Virtuoso endpoint. Além disso, o processo de construção da resposta também usa padrões pré-definidos. A Figura 1 mostra o workflow realizado pelo MediBot durante este modo. Sete tipos de consultas foram definidos, conforme mostrado na Tabela 1.

\footnotetext{
${ }^{1}$ https://anonimoanim10.github.io/mediBot/
} 
Em complemento ao modo resposta rápida, MediBot possui um modo interativo. Enquanto o primeiro fornece acesso rápido e fácil às informações, o segundo fornece uma forma versátil de acesso ao conhecimento contido no LDM.

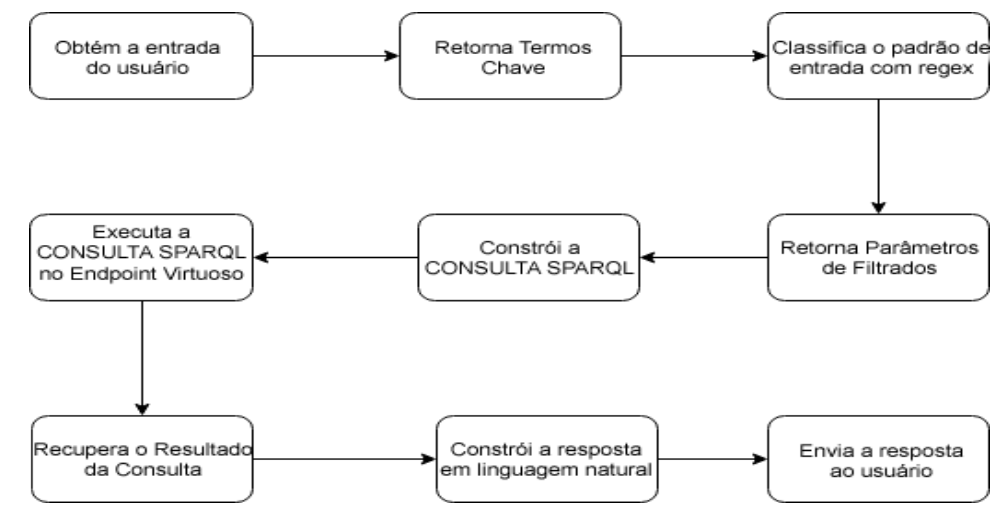

Figura 1. MediBot workflow.

Tabela 1. Tipos de consultas respondidas pelo MediBot no modo respostas rápidas.

\begin{tabular}{|l|l|}
\hline Tipo de Consulta & Exemplo \\
\hline Medicamentos com um princípio ativo & $\begin{array}{l}\text { Quais são os medicamentos com a } \\
\text { substância dipirona? }\end{array}$ \\
\hline Definição de termos no domínio & Defina Classe Terapêutica \\
\hline Informações sobre um certo medicamento & Fale sobre o medicamento aspirina \\
\hline Riscos de um medicamento & Quais são os riscos de um medicamento reopro? \\
\hline Lista de representações de um medicamento & Quais são as apresentações do medicamento reopro? \\
\hline $\begin{array}{l}\text { Informações sobre a apresentação do código } \\
\text { de barra }\end{array}$ & $\begin{array}{l}\text { Me informe sobre a apresentação do } \\
\text { código de barras 7896382701801 }\end{array}$ \\
\hline $\begin{array}{l}\text { Preço de uma apresentação com a taxa de } \\
\text { ICMS em um Estado }\end{array}$ & $\begin{array}{l}\text { Quais são os preços com taxa de ICMS } \\
\text { da apresentação 7896382701801 } \\
\text { no estado do Ceará? }\end{array}$ \\
\hline
\end{tabular}

Durante o modo interativo, dois tipos de tarefas podem ser executadas, navegar e consultar. Para a decisão de quais próximos passos devem ser seguidos durante a conversação em uma tarefa específica MediBot usa informações sobre interações passadas durante a mesma tarefa (contexto), o ponto atual da tarefa (state) e, nos casos em que o chatbot espera uma resposta do usuário, é recebida uma mensagem (input). A seguir são descritas as tarefas:

- Tarefa de Consulta: Permite ao usuário consultar e visualizar os dados sobre instâncias contidas na base de conhecimento, mostrando valores de atributos de instâncias. A tarefa de consulta é recursivamente interativa. Ao receber uma consulta de um usuário, MediBot retorna uma lista de propriedades da instância, dando ao usuário a opção de selecionar uma destas para visualizar seus valores. Na Figura 2 é apresentado um exemplo de interação da tarefa de consulta através do Telegram.

- Tarefa de Navegação: Permite ao usuário navegar recursivamente através dos termos existentes na base de conhecimento (knowledge base), mostrando definições e esquema da fonte. Quando o usuário inicia a tarefa de navegação sobre um termo, MediBot apresenta diferentes nomes, tipos e definições de termo, além de apresentar suas propriedades e permitir ao usuário selecionar uma destas para ser o novo pivô da tarefa, podendo navegar sobre os conceitos definidos na ontologia. Para iniciar esta tarefa basta digitar a palavra "Explore". 


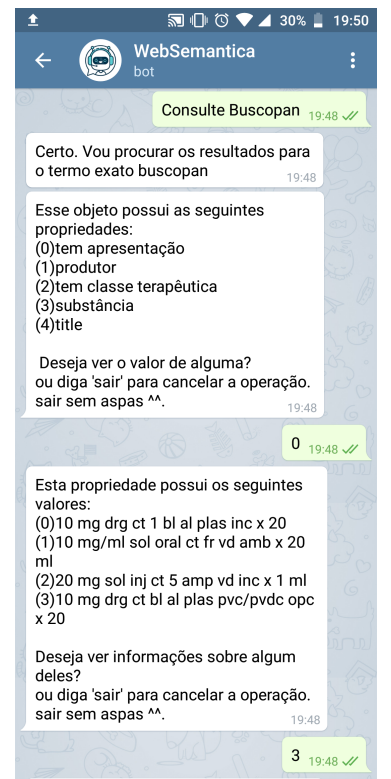

(a) Tarefa Consulta

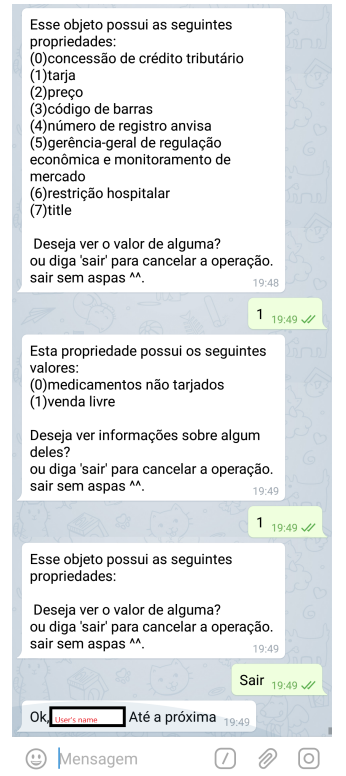

(b) Tarefa Consulta

Figura 2. Exemplos de Consulta no Telegram

\section{Arquitetura}

A arquitetura do MediBot é organizada em três camadas: $i$ ) interface de usuário; ii) servidor e iii) conhecimento. A Figura 3 apresenta a arquitetura do MediBot e seus componentes.

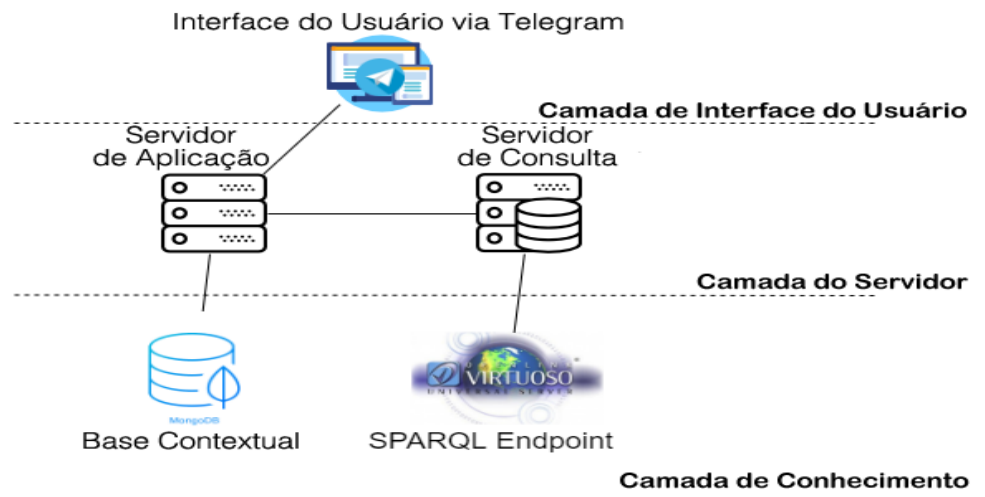

Figura 3. Arquitetura MediBot

Na primeira camada, a interface do usuário fornece um meio de interação com o usuário através do aplicativo de mensagens instantâneas Telegram. A utilização do MediBot pode ser feita através do aplicativo móvel do Telegram ,ou, por seu aplicativo desktop no computador. A razão para escolher o Telegram como um canal para o MediBot se dá em razão dessa ferramenta já possuir uma grande base de usuários em conjunto com uma infraestrutura robusta, além de ter uma $A P I$ fácil que permite a criação e uso de chatbots.

A segunda camada proposta é a camada do servidor. Esta camada é responsável por processar as requisições e prover respostas, sendo composta por dois componentes 
principais. O primeiro componente desta camada é o servidor de aplicação (application server), responsável por receber e processar as requisições do usuário. O application server recebe a requisição do usuário e constrói a consulta SPARQL responsável por retornar a informação desejada. Ainda, tal componente é também responsável por enviar a consulta SPARQL para o servidor de consultas (query server) e emitir uma mensagem de confirmação de entrega ao usuário. Sendo o segundo componente, o query server é responsável por receber a consulta SPARQL e executá-la no SPARQL endpoint. Além disso, esse componente também é responsável por obter a resposta da consulta SPARQL e construir a mensagem de resposta ao usuário.

A terceira e última camada é a camada de conhecimento. Esta camada é responsável por armazenar o conhecimento necessário para interagir e responder o usuário. Esta camada também é composta por dois componentes:

- Base Contextual: Responsável por armazenar informações relevantes ao contexto, tais como informações pessoais sobre o usuário como seu nome, sobrenome, linguagem e outras informações referentes ao fluxo de diálogo. As informações sobre o contexto da interação são armazenadas em uma instância no banco de dados não-relacional orientado a objetos mongoDB, que permite um rápido armazenamento e recuperação de dados armazenados em JSON, permitindo o processamento direto por código javascript sem a necessidade de pré-processamento dos dados. Os links para as fontes originais podem ser encontrados em ${ }^{2}$.

- Linked Data Mashup (LDM): O processo de Linked Data Mashup foi realizado com os dados de medicamentos presentes nas bases da Agência Nacional de Vigilância Sanitária (ANVISA) e da base SIDER presente no projeto BIO2RDF [Belleau et al. 2008]. Da ANVISA, foram selecionados:

- Preços de Medicamentos ao Consumidor (PMC);

- Preços de Medicamentos ao Governo (PMG);

- Riscos do medicamento em gestantes e lactantes (RGL);

PMC e PMG são encontrados nos formatos de arquivo $X L S$ e $P D F$, sendo usadas neste trabalho as versões XLS. Ambos os datasets contêm informações sobre medicamentos alopáticos, como o nome do medicamento, o produtor, o código de barras, a classe terapêutica, a apresentação, o princípio ativo e os preços. O dataset RGL contém as categorias de risco de substâncias durante o período da gravidez e amamentação. Este dataset só está disponível no formato não estruturado $P D F$. Por sua vez, o dataset do SIDER disponibilizado pelo projeto BIO2RDF já está no formato $R D F$. O dataset SIDER contém dados sobre drogas, suas indicações, efeitos colaterais e diferentes rótulos. No entanto, o banco de dados só possui dados em inglês, não contendo informações sobre medicamentos brasileiros, tornando necessário traduzi-los para o português. Este dataset foi selecionado porque contém informações sobre os efeitos colaterais dos princípios ativos, tais informações são necessárias para informar os riscos de um medicamento.

Por fim, foi gerado um Linked Data Mashup (LDM) representando uma visão integrada das múltiplas fontes seguindo um mesmo vocabulário e método de armazenamento e acesso, além dos arquivos de mapeamento e da implementação OWL da ontologia, podendo ser acessados publicamente via datahub ${ }^{3}$. O LDM

\footnotetext{
${ }^{2}$ https://anonimoanim10.github.io/mediBot/\#fontes

${ }^{3} \mathrm{https}: / /$ datahub.io/linkeddatamashupeducacional/data-med/v/2
} 
resultante foi hospedado no virtuoso triplestore, que é acessado diretamente pelo aplicativo MediBot através do servidor de consulta.

\section{Demostração proposta}

Para a demostração da efetividade da ferramenta MediBot como uma ferramenta de apoio a recuperação de informação, propomos o seguinte cenário:

- Um usuário busca saber quais são os riscos de um determinado medicamento através do modo de respostas rápidas.

- Posteriormente o usuário busca por medicamentos que possuem o mesmo princípio ativo. Esta atividade pode ser realizada tanto através do modo rápido, quanto por meio do modo de consulta.

- Ao ver termos como tarja, tipo de medicamento, classe terapêutica, etc. O usuário seleciona a opção no qual deseja, levando-o a navegar sobre os termos da ontologia e posteriormente obtendo informações.

\section{Considerações Finais}

A automedicação sem instrução prévia é uma problemática que vem apresentando indicadores preocupantes no que tange a intoxicação. Neste trabalho foi apresentado MediBot, um chatbot para consulta de riscos e informações sobre medicamentos. Para tanto, $M e$ diBot apresenta-se como um instrumento interativo para auxílio no ato de recuperação de informações sobre medicamentos e seus riscos, possibilitando uma redução em relação às eventuais dúvidas do paciente.

Como trabalhos futuros, pretende-se disponibilizar MediBot para outras plataformas de mensagem instantânea, como Facebook Messenger e Instagram Direct, além de expandir o conjunto de padrões de consultas rápidas.

\section{Referências}

Belleau, F. et al. (2008). Bio2rdf: towards a mashup to build bioinformatics knowledge systems. Journal of biomedical informatics, 41(5):706-716.

Bizer, C. et al. (2011). Linked data: The story so far. In Semantic services, interoperability and web applications: emerging concepts, pages 205-227. IGI Global.

Corrêa, A. et al. (2013). Uma abordagem sobre o uso de medicamentos nos livros didáticos de biologia como estratégia de promoção de saúde. Ciência \& Saúde Coletiva, 18:3071-3081.

Shadbolt, N. et al. (2006). The semantic web revisited. IEEE intelligent systems, 21(3):96-101.

SINITOX (2016). Sistema Nacional de Informações Toxicológicas registro de intoxicações no brasil. Acessado: 10-02-2019.

WHO et al. (2002). Safety of medicines: a guide to detecting and reporting adverse drug reactions. Technical report, Geneva: World Health Organization. 